\title{
The influence of a slider gap in the beam-slider structure with an MFC element on energy harvesting from the system: experimental case
}

Received: 17 April 2020 / Revised: 16 September 2020 / Accepted: 23 October 2020 / Published online: 23 December 2020

(C) The Author(s) 2020

\begin{abstract}
A passively self-tuning resonator configuration is presented in this study. Under certain operating conditions, a self-resonating system has the capability to passively adjust dynamical characteristics until the whole system becomes resonant. A clamped-clamped beam with an attached mass sliding along the beam and a slight gap that, under a harmonic input excitation and well-defined operating regime, can lead to the increase in voltage amplitude generated by the piezo-harvester attached to this structure may be an example of such a system. Taking into account such behavior of the system, the paper is focused on determining the distributedparameters of the electromechanical system versus a different slider position on the beam in modal coordinates. The obtained simulation results, considering the homogenous model of an MFC element for the desired slider locations, showed how the width of the gap between the slider and the beam additionally influences the voltage generated by the piezo-harvester. Experimental tests carried out on the real stand with an EHE301 module and the designed SSHI interface circuit allowed to verify the numerical results and also showed the influence on the resistive load connected to the system for an improvement of the considered energy harvesting system parameters.
\end{abstract}

\section{Introduction}

Vibration energy harvesting has been a research topic of growing interest over the past two decades due to the simultaneous increase in the demand of wireless sensing capabilities and the decrease in power requirements of radio transmitters, sensors, and logic controllers [1-6]. Customized frequency operation of the harvesting element to the excitation frequency of the input is a barrier that should be overcome to succeed in resonant vibration energy harvesting. Such behavior can be visible in real mechanical structures like motors, fans, compressors, side panels of public busses, industrial machines or other applications with an operational frequency that is not homogenous where, as it is known, sources of vibrations change in frequency over time [7, 8]. Additionally, the vibration frequency of a machine is rarely known a priori, so it requires customizing the energy harvesters for each machine. In some modern applications, it is highly desirable to have energy harvesters that passively tune their resonance frequency to match the input vibration frequency without requiring precious power to be siphoned off for an active feedback control system and without requiring customization at the time of installation.

The problem of frequency mismatch in vibration energy harvesters is solved by using active and passive methods. In the first one, it is solved by designing a stiffness control system of chosen mechanical structures or the system mass control by using an externally supplied force or an electronic signal to change the resonance of the energy harvester [8]. However, in another case, it is realized without any additional external energy applied

\footnotetext{
A. Koszewnik $(\varangle)$

Department of Robotics System and Mechatronics, Bialystok University of Technology, Wiejska Street 45C, 15-351 Białystok, Poland

e-mail: a.koszewnik@pb.edu.pl
} 
to the system. Regardless of the choice of the aforementioned methods, it is known from the literature that the square of resonance frequency is directly proportional to the stiffness and inversely proportional to the mass. Due to this dependence, the effect of customizing the frequency of the system to the natural frequency of the harvester can be achieved by using the frequency modification strategies or broadband techniques. The first one was described in [2, 3, 9]. In [9], the authors presented the beam in terms of the linear Euler-Bernoulli theory and next proposed a model of a beam-slider system. In this case, the slider was modeled as a rigid body and assumed to be vertically constrained to the beam, which corresponds to a tight fit without clearance. Moreover, taking into account experimental results, these authors observed that the beam vibrational deflection shape had only one antinode at the beam center. This leads to the conclusion that the slider moves to the antinode of the first vibrational deflection mode shape, and the slider moves toward the beam center and stays there. In another paper [10], a similar effect has been analyzed by Krack et al. This group of researchers refined the Miller model from [9] by taking into account a small clearance between the slider and the beam. It was also shown that frictional contact interactions are responsible for the movement of the slider away from the beam center and in the case of a too tight fit (small clearance between the slider and the beam); this slider never moves away from the center, in contrast to the experimental observations. As a result, they concluded that the self-adaption acts for a broad, yet limited, range of excitation frequencies around the first natural frequency of the system.

In the case of broadband techniques, this problem is solved by applying an additional mechanical stopper introducing nonlinearities [7, 11-15] or considering a magnetic field induced between a stationary magnet and a magnet attached to the harvester beam [8, 16-19]. In [11], experimental investigations of the beam-slider structure were performed. The obtained experimental results showed that this structure could achieve selftuning and maintain its tuned state as a frequency sweep up and down, even though the device was scaled down. Similar experimental investigations of the self-tuning behavior of a structure of this kind have also been shown by Pillatstch et al. in [13, 14]. In both papers, the authors observed a discrepancy existing between the beam displacement and the output voltage, especially when piezoelectric patches were bonded to the clamped-clamped beam. They also showed that the characteristics of the beam-slider structure were sensitive to parameters such as mass and gap size. The parameters of an energy harvesting system located on the beam-slider system were also investigated by a group of researchers from Japan [19]. In this manuscript, numerical and experimental studies of the effect of weight on the resonant tuning and energy harvesting characteristics of energy harvesting devices using giant magnetostrictive materials were described. In the results of these investigations, the authors indicated that this kind of a mechanical system can be used to design progressive vibration energy harvesting devices that do not require an external power supply to adjust their resonant frequency to the input vibration.

Taking into account the showed peer review, this paper is focused on the analysis of an electromechanical model of a piezo-harvester located on a beam-mass system. In order to enhance the efficiency of the energy harvesting system, the considered mechanical system was equipped with a slider that allows to adjust the frequency of the system to the frequency resonance of the harvester and investigate self-resonance behavior of the whole system. Additionally, the performed analysis of the distance between the slider and the beam became a supplement of previously published results. Next, taking into account the proposed solution, the paper is organized as follows. Section 2 presents distributed-parameters of the self-resonance electromechanical system in the modal coordinates determined on the basis of the analytical approach. Section 3 presents the FEM model of the beam-slider structure with a homogenous model of MFC elements to enhance vibration-based energy harvesting systems. As a result, this is a novelty part of this manuscript. Additionally, numerical results allowed to verify the theoretical results presented in the previous Section. In the next Section, experimental investigations using an EHE001 module and the synchronized switched harvesting on the inductor (SSHI) circuit have been presented and described [20,21]. It allows to show the novelty of this manuscript. Also, this approach allows to check proper module conditioning and assess the size of the gap in the system to generate optimal energy from this kind of sources. Section 5 concludes the main findings of this work.

\section{Electromechanical model of a self-resonating energy harvester}

The energy harvesting system considered in this paper consists of a rigid slider moving freely along the clamped-clamped aluminum beam. To harvest energy, a macro-fiber composite (MFC) stripe element was attached to the beam, and next it was mounted on the vibration shaker providing a harmonic excitation. As a result, it led to obtaining a smart structure, which is shown in Fig. 1 where parameters of particular elements (the beam, the slider, and the piezo-harvester) are collected in Table 1. 
(a)

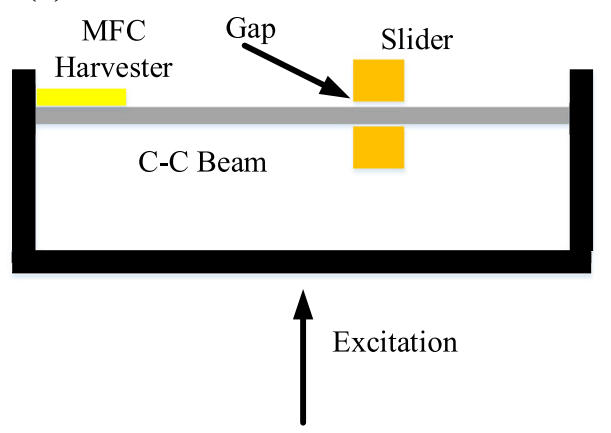

(b)

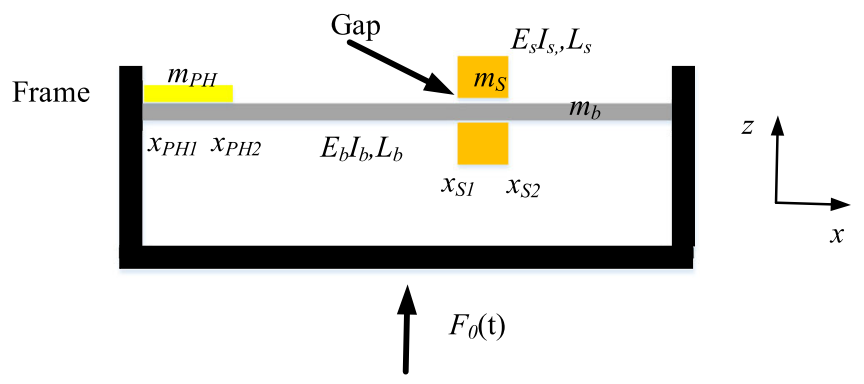

Fig. 1 The scheme of the beam-mass resonator system with a free slider and the MFC piezo-harvester element

Table 1 Parameters of the host structure, the slider, and the piezo-element [22, 24]

\begin{tabular}{|c|c|c|c|c|c|c|}
\hline \multirow{2}{*}{$\begin{array}{l}\text { Parameter } \\
\text { Material }\end{array}$} & \multicolumn{2}{|c|}{$\mathrm{C}-\mathrm{C}$ beam } & \multicolumn{2}{|c|}{ Slider } & \multicolumn{2}{|c|}{$\begin{array}{l}\text { Piezo-harvester MFC } \\
8528 \text { P2 }\end{array}$} \\
\hline & \multicolumn{2}{|c|}{ Aluminum } & \multicolumn{2}{|c|}{ Aluminum } & \multicolumn{2}{|c|}{$\begin{array}{l}\text { MFC macro-fiber } \\
\text { composite }\end{array}$} \\
\hline Length (m) & $L_{\mathrm{b}}$ & 0.38 & $L_{\mathrm{s}}$ & 0.030 & $L_{\mathrm{PH}}$ & 0.056 \\
\hline Width (m) & $h_{\mathrm{b}}$ & 0.028 & $h_{\mathrm{s}}$ & 0.060 & $h_{\mathrm{PH}}$ & 0.028 \\
\hline Thickness (m) & $t_{\mathrm{b}}$ & 0.002 & $t_{\mathrm{s}}$ & - & $t_{\mathrm{PH}}$ & $300 \mathrm{e}-6$ \\
\hline Density $\left(\mathrm{kg} / \mathrm{m}^{3}\right)$ & $\rho_{\mathrm{b}}$ & 2700 & $\rho_{\mathrm{s}}$ & 2700 & $\rho_{\mathrm{PH}}$ & 1500 \\
\hline Young's modulus (GPa) & $E_{\mathrm{b}}$ & 69 & $E_{\mathrm{S}}$ & - & $E_{\mathrm{PH}}$ & 9.5 \\
\hline Poisson's ratio & - & 0.33 & - & 0.3 & - & 0.33 \\
\hline Piezoelectric strain constant (pC/N) & - & - & - & - & $d_{31}$ & -173 \\
\hline Piezoelectric stress/charge constant $\left(\mathrm{C} / \mathrm{m}^{2}\right)$ & - & - & - & - & $e_{33}$ & 1600 \\
\hline
\end{tabular}

The clamped-clamped beam with a slider and an integrated harvester is excited to vibration by force $F_{0}$ from the shaker at the center point of the beam. Then, the transverse vibration general equation of this structure takes the following form [23]:

$$
\begin{aligned}
& {\left[E I+E_{\mathrm{PH}} I_{\mathrm{PH}}\left(H\left(x-x_{\mathrm{PH} 2}\right)-H\left(x-x_{\mathrm{PH} 1}\right)\right)\right] \frac{\partial^{4} w(x, y)}{\partial x}+\left[m_{\mathrm{b}}+m_{\mathrm{S}}\left(H\left(x-x_{S 2}\right)-H\left(x-x_{S 1}\right)\right)+\cdots\right.} \\
& \left.m_{\mathrm{PH}}\left(H\left(x-x_{\mathrm{PH} 2}\right)-H\left(x-x_{\mathrm{PH} 1}\right)\right)\right] \frac{\partial^{2} w(x, y)}{\partial x}-\Gamma V(t)\left[\frac{\mathrm{d} \partial\left(x-x_{\mathrm{PH} 2}\right)}{\mathrm{d} x}-\frac{\mathrm{d} \partial\left(x-x_{\mathrm{PH} 1}\right)}{\mathrm{d} x}\right]=F_{0} \delta\left(x-x_{0}\right)
\end{aligned}
$$

where $E$ denotes Young's modulus of the beam and the slider, $E_{\mathrm{PH}}$ Young's modulus of the MFC harvester, $I$ the inertia moment of the beam, $I_{\mathrm{PH}}$ the inertia moment of the MFC harvester, $m_{\mathrm{b}}$ the mass per unit length of the beam, $m_{\mathrm{S}}$ the mass per unit length of the slider, $m_{\mathrm{PH}}$ the mass per unit length of the MFC harvester, $H(x)$ the Heaviside function, $\delta(x)$ the Dirac function along the $X$ axis, $V(t)$ the voltage flowing through the external resistive load $R, F_{0}$ the force derived from the vibration shaker, and $\Gamma$ the electromechanical coupling term.

The modal value of the electromechanical coupling term $\tilde{\Gamma}_{n}$ mentioned in Eq. (1) of the system is described as:

$$
\tilde{\Gamma}_{n}=-E_{\mathrm{PH}} d_{31} w_{\mathrm{PH}} t_{c}
$$

where $t_{c}$ denotes the thickness between the neutral axes of the beam and the piezo-harvester, $t_{c}=\frac{t_{\mathrm{b}}}{2}+\frac{t_{\mathrm{PH}}}{2}$, $d_{31}$ the piezoelectric strain coefficient of the MFC element, $w_{\mathrm{PH}}$ the width of the MFC harvester, and $t_{\mathrm{PH}}$ the thickness of the MFC harvester.

The piezoelectric harvester integrated with the host structure requires also considering the approach from the electrical point of view. For this purpose, the electrical charge accumulated at its electrodes can be calculated over the whole active surface area in the following form $[2,5]$ :

$$
Q=\int_{x_{-} \mathrm{PH} 1}^{x_{-} \mathrm{PH} 2}\left(d_{31} E_{\mathrm{PH}} \bar{\delta}_{\mathrm{PH}}+\varepsilon_{33} E_{3}\right) w_{\mathrm{PH}} \mathrm{d} x
$$


where $\varepsilon_{33}$ represents the permittivity at constant stress, $\bar{\delta}_{\mathrm{PH}}$ the bending strain along the middle surface of the piezo-layer, $E_{3}$ the electric field, $x_{-} \mathrm{PH} 1$ the beginning location of the MFC harvester on the host structure, and $x_{-}$PH2 the ending location of the MFC harvester on the host structure.

Next, with Ohm's law being applied, the current flowing through the load resistor $R$ can be expressed as:

$$
i(t)=\frac{V(t)}{R}=\frac{\mathrm{d} Q}{\mathrm{~d} t}=\frac{\mathrm{d}}{\mathrm{d} t}\left[\int_{x_{-} \mathrm{PH} 1}^{x_{-} \mathrm{PH} 2}\left(d_{31} E_{\mathrm{PH}} \bar{\delta}_{\mathrm{PH}}+\varepsilon_{33} E_{3}\right) w_{\mathrm{PH}} \mathrm{d} x\right]
$$

where $R$ denotes the resistive load applied to the system.

Taking into account Eq. (4), it can be seen that the current $i(t)$ is strongly associated to strains of the piezoelectric harvester and the electrical field applied to its electrodes. Because of that, the electrical circuit of the system can be obtained by substituting the electric field $E_{3}=-V(t) / t_{\mathrm{PH}}$ and the strain $\bar{\delta}_{\mathrm{PH}}=-t_{c} \frac{\partial^{2} w}{\partial x^{2}}$ in the following form [13]:

$$
C_{\mathrm{p}} \frac{d V(t)}{d t}+\frac{V_{\mathrm{p}}}{R}+\Gamma\left[\int_{x_{-} \mathrm{PH} 1}^{x_{\mathrm{PH} 2}} \frac{\partial^{3} w(x, t)}{\partial x^{2} \partial t} \mathrm{~d} x\right]=0
$$

where $C_{\mathrm{p}}$ represents the capacitance of the MFC harvester and $C_{\mathrm{p}}=\frac{\varepsilon_{33} w_{\mathrm{PH}} l_{\mathrm{PH}}}{t_{\mathrm{PH}}}$.

Equations (1) and (4) describe the electro-elastic model of the MFC harvester integrated with the beam in physical coordinates. However, from the energy harvesting point of view, further analysis of this system should be also performed in modal coordinates. Consequently, the vertical displacement of the considered host structure can be represented in the following form:

$$
w(x, t)=\sum_{n=1}^{\infty} \phi_{n}(x) \eta_{n}(t)
$$

where $\phi_{n}(x)$ denotes the mass normalized eigenfunction (mode shapes) and $\eta_{n}(t)$ the modal coordinate of the considered frame for the $n$th mode.

Taking into account Fig. 1, the clamped-clamped beam is the mechanical structure. Because of that, eigenvectors of such a beam, after considering the boundary coordinates, given in Eq. (6), and split by geometric and time variables, can be expressed as [23]:

$$
\begin{gathered}
w(0, t)=0,\left.\frac{\partial w(x, t)}{\partial x}\right|_{x=0}=0, \quad w\left(L_{\mathrm{b}, t}\right)=0,\left.\quad \frac{\partial w(x, t)}{\partial x}\right|_{x=L_{\mathrm{b}}}=0, \\
\varphi_{n}(x)=\left(\cosh \left(\lambda_{n} x\right)-\cos \left(\lambda_{n} x\right)\right)+\sigma_{r}\left(\sinh \left(\lambda_{n} x\right)-\sin \left(\lambda_{n} x\right)\right)
\end{gathered}
$$

where $\lambda_{n}=\frac{(2 n+1) \pi}{2}-$ denotes the frequency parameter of an undamped host structure, while $\sigma_{r}=$ $\frac{\sin \left(\lambda_{n}\right)-\sinh \left(\lambda_{n}\right)+\lambda_{n} \frac{m_{\mathrm{s}} d_{m}}{m_{\mathrm{b}} L_{\mathrm{b}}}\left(\cos \left(\lambda_{n}\right)-\cosh \left(\lambda_{n}\right)\right)}{\cos \left(\lambda_{n}\right)+\cosh \left(\lambda_{n}\right)-\lambda_{n} \frac{m_{\mathrm{s}} d_{m}}{m_{\mathrm{b}} L_{\mathrm{b}}}\left(\sin \left(\lambda_{n}\right)-\sinh \left(\lambda_{n}\right)\right)}$ wherein $d_{m}$ is the distance from the center of the beam's cross-sectional area to the tip mass' center of mass.

Equation (7) put into Eq. (1) leads to solving the eigenvalue problem of the smart beam for short circuit conditions. Then, the natural frequency $\omega_{n}$ of the structure without the patch MFC harvester for the $n$-th mode can be presented in the following form:

$$
\omega_{n}=\lambda_{n}^{2} \sqrt{\frac{E I}{m_{\mathrm{b}} L_{\mathrm{b}}^{4}}}
$$

where $L_{\mathrm{b}}$ denotes the length of the beam.

Next, taking into account the modal analysis procedure of the beam structure with a piezo-harvester adjusted to its surface by using support, an electromechanical coupled ordinary differential equation for the modal time response $\eta_{n}$ can be expressed as [2]:

$$
\frac{\mathrm{d}^{2} \eta_{n}(t)}{\mathrm{d} t^{2}}+2 \xi_{n} \omega_{n} \frac{\mathrm{d} \eta_{n}(t)}{\mathrm{d} t}+\omega_{n}^{2} \eta_{n}(t)+\tilde{\Gamma}_{n} v(t)=f_{n}(t) \delta\left(x-x_{0}\right)
$$


where $\xi_{n}$ is the modal damping ratio and is $f_{n}(t)$ the modal force applied to the structure.

As a result, the modal electromechanical coupling term $\tilde{\Gamma}_{n}$ can be represented as:

$$
\tilde{\Gamma}_{n}=-E_{\mathrm{PH}} d_{31} w_{\mathrm{PH}} t_{c} \int_{x_{\mathrm{PH} 1}}^{x_{\mathrm{PH} 2}} \frac{\mathrm{d}^{2} \phi_{n}(x)}{\mathrm{d} x^{2}} \mathrm{~d} x=-\left.E_{\mathrm{PH}} d_{31} w_{\mathrm{PH}} t_{c} \frac{\mathrm{d} \phi_{n}(x)}{\mathrm{d} x}\right|_{x_{-} \mathrm{PH} 1} ^{x_{-} \mathrm{PH} 2} .
$$

The obtained modal electromechanical coupling term, given by Eq. (11), and the vertical deflection from Eq. (7) put into Eq. (4) lead to modifying the electrical circuit equation in the following form:

$$
C_{\mathrm{p}} \frac{\mathrm{d} V(t)}{\mathrm{d} t}+\frac{V}{R}-\sum_{n=1}^{\infty} \tilde{\Gamma}_{n} \frac{\mathrm{d} \eta_{n}(t)}{\mathrm{d} t}=0 .
$$

The performed considerations of the clamped-clamped beam with an integrated MFC patch harvester for modal coordinates allow to calculate the value of the rectified voltage response accumulated on the resistor $R$ in the steady state. For this purpose, assuming the harmonic form of the force formed as $f(t)=F_{0} \sin (\omega t)=F_{0} \mathrm{e}^{j \omega t}$, the modal voltage response $V(\mathrm{t})$ and the modal response of the system $\eta_{n}$ can be also expressed in the following forms:

$$
V(t)=\operatorname{Re}\left\{\tilde{V}_{n} \mathrm{e}^{j \omega t}\right\}, \quad \eta_{n}=\operatorname{Re}\left\{\tilde{\eta}_{n} \mathrm{e}^{j \omega t}\right\} .
$$

Substituting Eq. (13) into Eq. (10) and Eq. (12) leads to modifying the electromechanical model of the electrical circuit system to the following form:

$$
\begin{gathered}
-\omega^{2} \tilde{\eta}_{n} \mathrm{e}^{j \omega t}+2 j \xi_{n} \omega_{n} \omega \tilde{\eta}_{n} \mathrm{e}^{j \omega t}+\omega_{n}^{2} \tilde{\eta}_{n} \mathrm{e}^{j \omega t}-\tilde{\Gamma}_{n} V \mathrm{e}^{j \omega t}=F_{0} \mathrm{e}^{j \omega t} \phi_{n}(x), \\
C_{\mathrm{p}} V j \omega \mathrm{e}^{j \omega t}+\frac{V \mathrm{e}^{j \omega t}}{R}+\mathrm{e}^{j \omega t} \sum_{n=1}^{\infty} j \tilde{\Gamma}_{n} \omega \tilde{\eta}_{n}=0 .
\end{gathered}
$$

Next, as a result of rejecting the harmonic part $\mathrm{e}^{j w t}$ from Eq. (14), the modal amplitude of the piezo-harvester response can be given by:

$$
\tilde{\eta}_{n}=\frac{\tilde{F}_{n}-\tilde{\Gamma}_{n} \tilde{V}}{\omega_{n}^{2}+2 j \xi_{n} \omega_{n} \omega-\omega^{2}}
$$

where

$$
\tilde{F}_{n}=F_{0} \phi\left(x_{0}\right) .
$$

Finally, the frequency response function of the modal voltage amplitude $V$ of the smart beam for various gaps between the slider and the beam in the case of substituting Eq. (16) into Eq. (15) in the frequency domain can be expressed in the following form [2,23]:

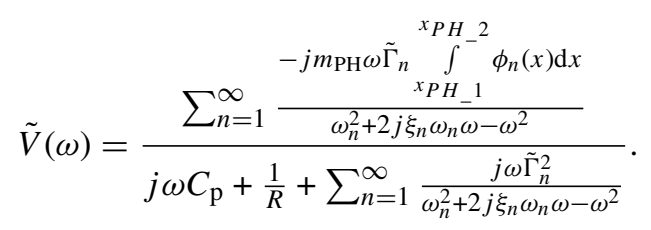

The theoretical results presented in Fig. 2 show that the size of the distance between the slider and the beam and the slider location have a significant influence on the voltage generated by the EH system. It is especially visible for the slider located in the distance of $100-130 \mathrm{~mm}$ from one fixed end of the beam where the indicated location is close to the MFC element. Then, the voltages generated from the EH system are highest, especially for the distance equaling $0.2 \mathrm{~mm}$. Finally, it can be concluded that the power output (order 2.5-6.4 mW) for these conditions should be also the highest but only in a narrow slider location range. 


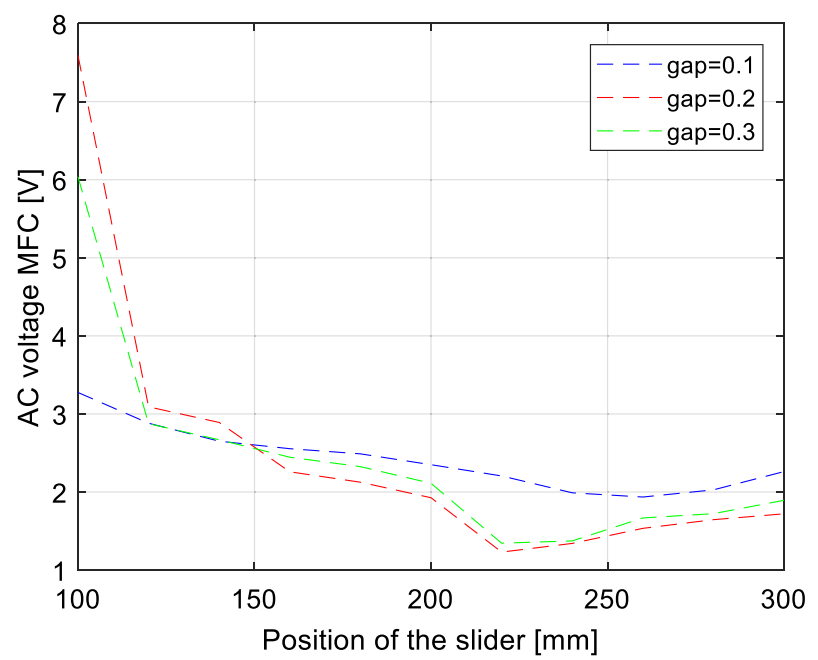

Fig. 2 The voltage generated by the macro-fiber composite for various locations of the slider on the beam

Table 2 Material properties of homogenized MFC layer of MFC8528 P2 [22]

\begin{tabular}{|c|c|c|c|c|c|c|c|c|c|}
\hline \multicolumn{2}{|c|}{$\begin{array}{l}\text { Young's modulus } \\
\text { (GPa) }\end{array}$} & \multicolumn{2}{|c|}{$\begin{array}{l}\text { Poisson's ratio } \\
(-)\end{array}$} & \multicolumn{2}{|c|}{$\begin{array}{l}\text { Shear modulus } \\
(\mathrm{GPa})\end{array}$} & \multicolumn{2}{|c|}{$\begin{array}{l}\text { Piezoelectric charge } \\
\text { coefficient }(\mathrm{pC} / \mathrm{N})\end{array}$} & \multicolumn{2}{|c|}{$\begin{array}{l}\text { Relative } \\
\text { permittivity (-) }\end{array}$} \\
\hline$E_{\mathrm{x}}$ & 31.6 & $v_{\mathrm{xy}}$ & 0.4 & $G_{\mathrm{xy}}$ & 4.9 & $d_{31}$ & -173 & $\varepsilon_{\mathrm{r}}^{\mathrm{T}}$ & 2253 \\
\hline$E_{\mathrm{y}}$ & 17.1 & $v_{\mathrm{yz}}$ & 0.2 & $G_{\mathrm{yz}}$ & 2.5 & $d_{32}$ & -150 & & \\
\hline$E_{\mathrm{z}}$ & 9.5 & $v_{\mathrm{xz}}$ & 0.4 & $G_{\mathrm{xz}}$ & 2.4 & $d_{33}$ & 325 & & \\
\hline
\end{tabular}

(a)

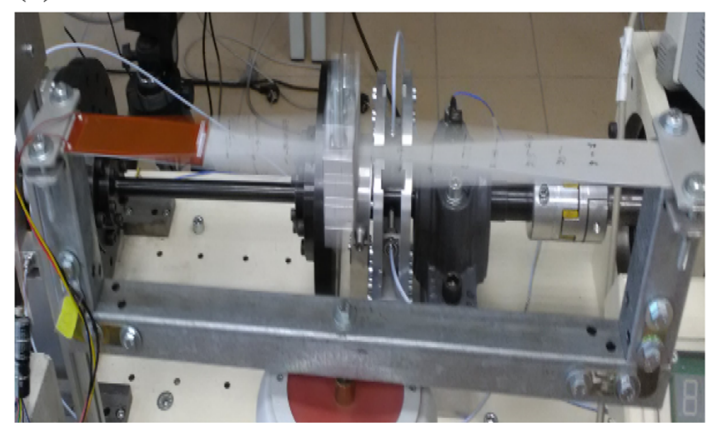

(b)

Fig. 3 a A photograph of a self-tuning system, b a numerical model of the beam-mass system with the MFC harvester

\section{Numerical analysis of a self-resonance beam with a piezo-patch harvester}

The numerical calculations of the slider-beam system with the MFC element are described in this Section. In order to assess parameters of a vibration-based energy harvesting $(\mathrm{EH})$ system and verify the theoretical results, the considered system was modeled in ANSYS software. In order to do it, the 3D coupled-field element SOLID 186 is used to mesh the beam and the slider volumes, while the SOLID 226 is used to mesh the MFC volume with the homogenized material. The properties of the homogenized MFC layer are listed in Table 2. Such modeling of these volumes permits to perform the modal analysis of the structure with a fixed slider in the desired location and assess the voltage values generated by this system with different sizes of the gap between the slider and the beam. Regardless of the type of analysis (modal or harmonic response), the aluminum beam being the host structure was modeled as a clamped-clamped beam, while the slider-as a tip mass. Figure 3 shows a photograph of a real structure (a) and the FEM model (b).

In the first step of the numerical investigations, the eigenvalue problem of such a modeled structure with the tip mass equaling $182 \mathrm{~g}$ is solved by using ANSYS software within the frequency range of 10-30 Hz, containing a fundamental frequency. In order to do this, eleven different locations of the slider omitting the 


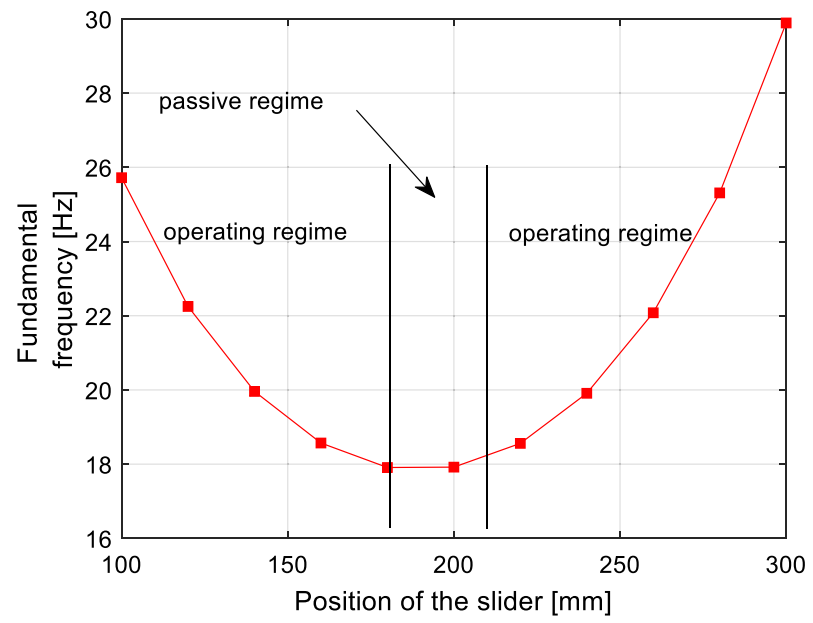

Fig. 4 The change of the fundamental frequency versus the slider location on the fixed-fixed beam

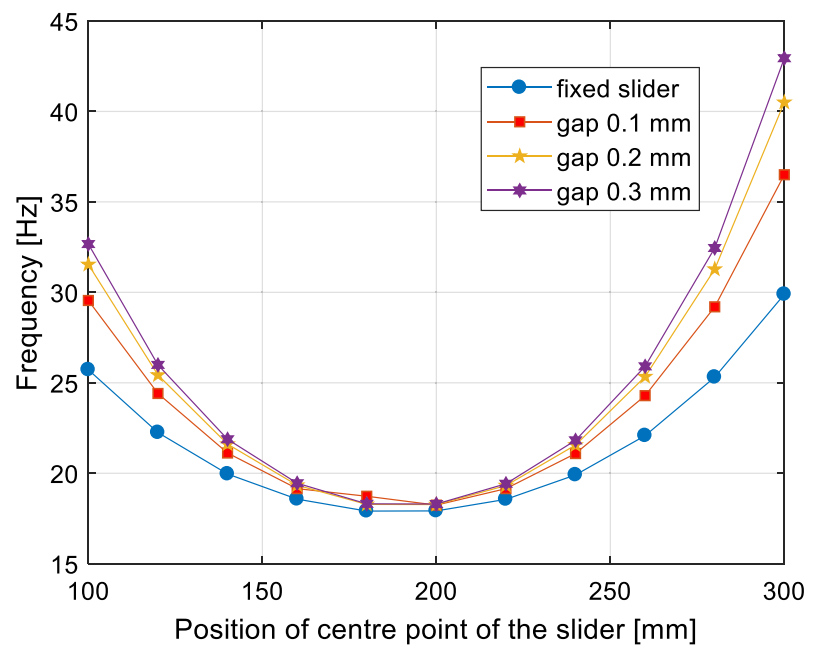

Fig. 5 The change of the fundamental frequency for different sizes of the gap between the slider and the beam versus the slider location on the clamped-clamped beam

harvester placement were chosen for further analysis in the range of 100-300 $\mathrm{mm}$ with a step equaling $20 \mathrm{~mm}$. This allowed to determine the modal parameters of the systems for a chosen location, and the results are presented in Fig. 4.

Taking into account Fig. 4, two operating regimes in which the value of the fundamental frequency is changed dynamically in relation toward the slider location on the host structure can be shown. The first operating regime was applied for the slider located within the range of $100-180 \mathrm{~mm}$, while the second one for the slider in the range of 220-300 mm. In other slider locations, a passive regime with a constant fundamental frequency was detected. Such behavior of the smart beam allowed to examine the influence of the gap size on the mechanical parameters of the system. In order to do this, numerical calculations were repeated for three different sizes of gaps: $0.1 \mathrm{~mm}, 0.2 \mathrm{~mm}$, and $0.3 \mathrm{~mm}$, respectively. The obtained results, shown in Fig. 5, indicate that the fundamental frequency of the system increases when the gap widens in both operating regimes, and it lightly increases for the passive regime. Consequently, this led to the conclusion that the process of assessing the energy harvesting system performances should be performed only for operating regimes, omitting the passive regime.

In the next step, strains of the considered system for different slider locations and the size of the gap were numerically analyzed. The proposed approach allowed to verify the analytical results and estimate the quasi-optimal location of the slider on the beam and the quasi-optimal size of the gap to obtain the maximum harvested energy from vibrations. In order to do this, the excitation force generated by an amplifier with a 


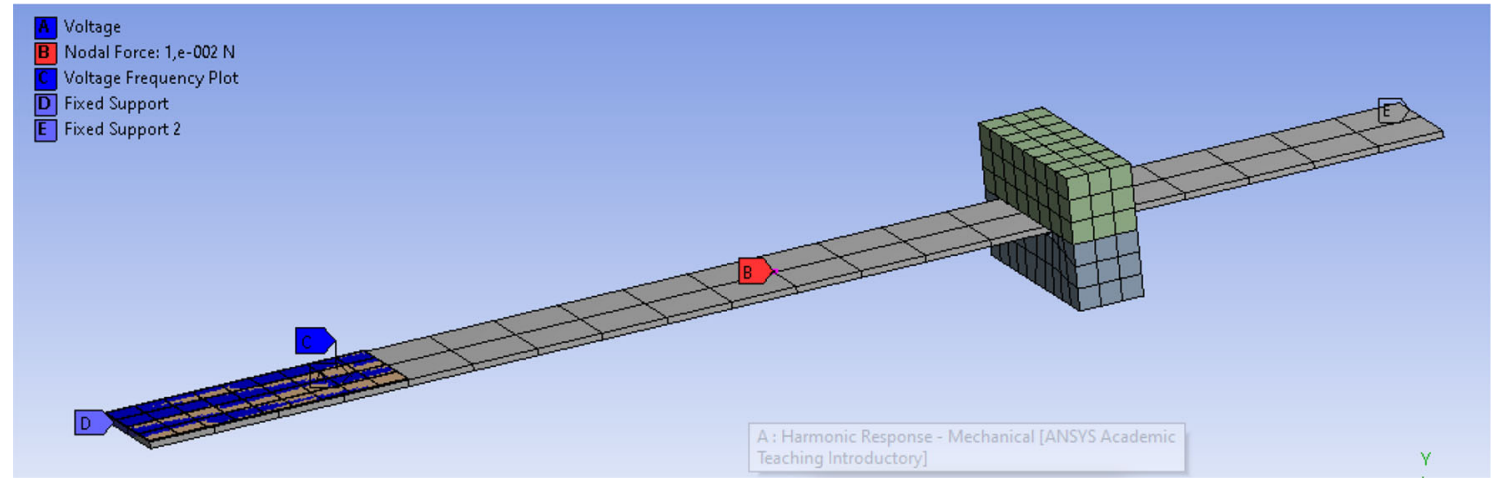

Fig. 6 The numerical model of a clamped-clamped beam with the freed slider and the applied sinusoidal force in the indicated nodes

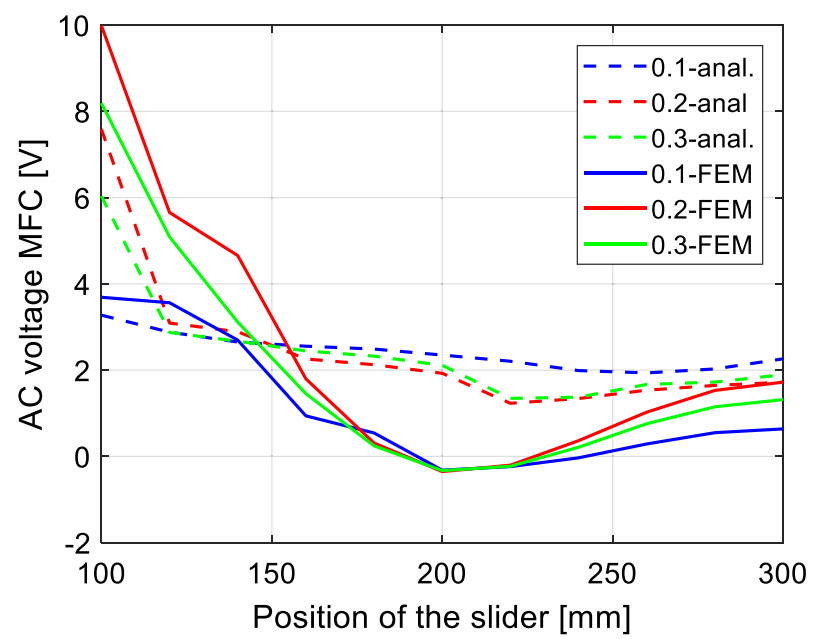

Fig. 7 Comparison of AC voltage generated by the MFC piezo-harvester located on the beam in the distance of 0-86 $\mathrm{mm}$ from the left fixed end for different positions of the slider in the range of 100-300 $\mathrm{mm}$ (solid line—numerical results, dash line—analytical results)

vibration shaker was defined in the form of a sinusoidal force applied to the beam in the vertical direction in the middle of the beam, as it is shown in Fig. 6.

In the next step, a proper location of the slider was analyzed. For this purpose, the slider placement was changed in the same way as in previous tests, but in this case for three different sizes of the gap: $0.1 \mathrm{~mm}$, $0.2 \mathrm{~mm}$ and $0.3 \mathrm{~mm}$, respectively. Moreover, the whole structure was excited to vibration with the frequency referring to the indicated slider location and the size of the gap. This led to calculating eleven different values of strains of the piezo-harvester and finally- $\mathrm{AC}$ voltages, whose value is proportionally associated with those strains.

The voltages presented in Fig. 7 show correlations between the analytical and the numerical results by considering different sizes of the gap, the slider position, and the voltage generated by the piezo-composite. In both cases, the highest power output was achieved for the distance between the slider and the beam equaling $0.2 \mathrm{~mm}$, and the slider was located very close to the MFC element. Further increase in this size (up to $0.3 \mathrm{~mm}$ ) led to obtaining the decreasing strains of the piezo-patch harvester, and, finally, to achieving smaller amplitudes of the generated voltages; taking this analysis into account, it can be concluded that a real system should also effectively generate the power output (order of several $\mathrm{mW}$ ) by maintaining the above assumptions: the size of the gap is in the range of $0.1-0.2 \mathrm{~mm}$, and the slider is located close to the piezo-harvester. 

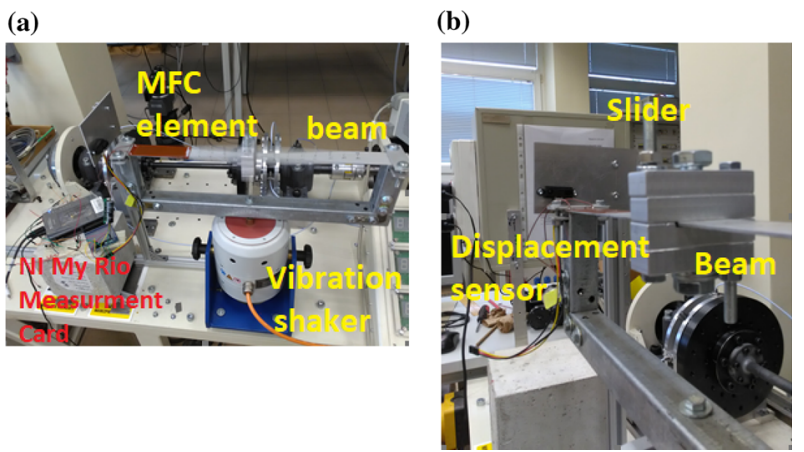

Fig. 8 a A photograph of the laboratory stand, $\mathbf{b}$ a photograph of the clamped-clamped beam with the slider, the MFC8528 P2 piezo-patch harvester, and the displacement sensor sharp

\section{Experimental setup and testing of the energy harvesting system located on a self-resonating beam}

In this Section, the performance of the energy harvesting system located on a self-resonating beam is tested on a laboratory stand (see. Fig. 8). In order to asses these performances, a passive beam with a slider was equipped with a piezo-patch sensor MFC8528 P2, developed by Smart Materials company, that was located very close to the left fixed end of the beam. Apart from these elements, the laboratory stand was retrofitted in the advanced linear conditioner EHE301 or the designed synchronized switch harvesting on inductor (SSHI) circuit interface (see Fig. 14) and the sharp displacement sensor used to measure voltages from the capacitor and the slider position on the beam toward its left fixed end, respectively.

Taking into account the scope of the experimental tests, the investigations were carried out in two stages. In the first one, the slider was located on the beam without a gap at the screw on the slider, while in the second one this slider was released, which caused its free movement along the beam. The freed slider allows to assess the parameters of the energy harvesting system for different sizes of the gap between the slider and the beam. Regardless of the slider type, the beam is excited to vibration in the same way each time by a harmonic signal generated from the signal generator developed by Agilent and next by applying it to the vibration shaker TIRA S-51110M with the amplifier BAA 120. On the other hand, from the measurement point of view, the slider position and both voltages from the conditioner system were measured and recorded by using an NI myRio 1900 measurement card connected also to the vibrating structure.

In the case of the fixed slider, investigations of the vibration-based energy harvesting system are carried out in the time domain by using a 3-axis Bruel \& Kjaer 4505 accelerometer with 5\% uncertainty (95\% confidence interval) according to the data provided by the manufacturer and of the digital signal analyzer (DSA). For this purpose, the harmonic signal in the form of a chirp signal $u(t)$ was applied to the vibration shaker by a TIRA signal amplifier in the frequency range of $1-120 \mathrm{~Hz}$. As a result, the vibration signal from the accelerometer was recorded for different positions of the slider on the beam changing in the range of 100-300 $\mathrm{mm}$ from one fixed end.

The obtained measurement signals of the vertical displacement of the structure were transformed to the frequency domain in order to verify the numerical results. Taking into account Fig. 9b, it can be seen that the highest peaks are obtained for the slider located close to both fixed ends of the beam, but the lowest-for the slider located in the middle of the beam. As a result, this properly verified the numerical results and indicated that the considered system works effectively in the frequency between 17 and $23 \mathrm{~Hz}$.

In the second step, the self-resonating behavior of the beam with the slider and MFC element was carried out for the freed slider with the gap of $0.1 \mathrm{~mm}$. In this case, the size of the gap was set by using a gap gauge. For this purpose, the smart structure was excited to vibration by applying a sinusoidal signal with the fundamental frequency corresponding to the desired position of the fixed slider. As a result, the free slider started moving to the position whose fundamental frequency equals the excitation frequency.

Taking into account this form of excitation, it can be seen that the behavior of the slider in relation to the vibrating beam is completely different, especially in the middle area part of the beam. Then, the ratio of the slider position with respect to a change of excitation frequency is very low. This led to omitting this area in further analysis and detecting two operating regimes where the size of the gap can affect the parameters of the EH system (Fig. 10). 

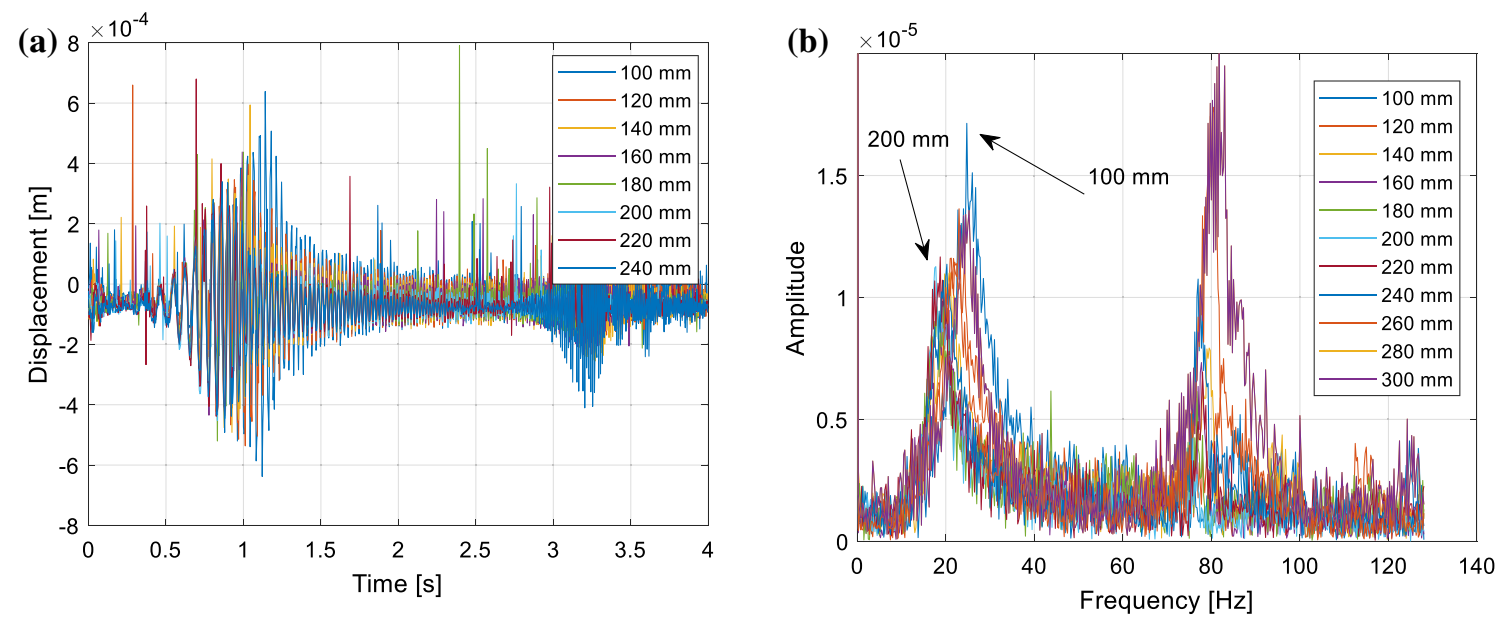

Fig. 9 a The recorded vibration signals of the smart structure with the slider in the time domain, $\mathbf{b}$ the vibration spectrum of the recorded signals for the desired slider location.

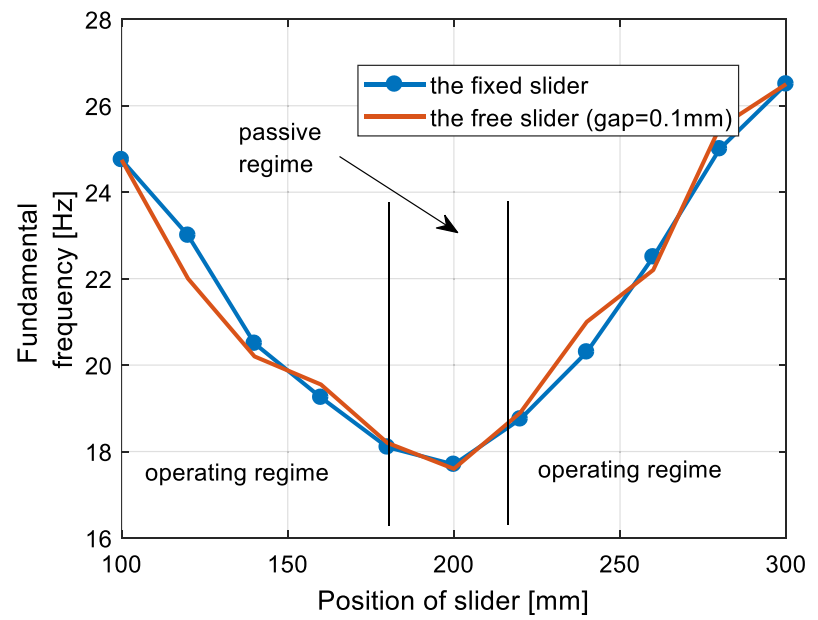

Fig. 10 The comparison of the fundamental frequencies of the smart beam and the MFC element for the fixed slider and the free slider

Next, the tested system with a gap of $0.2 \mathrm{~mm}$ was investigated in order to assess its ability to generate voltage from a vibrating piezo-stripe harvester for different slider locations. For this purpose, the slider was initially set in the operating regime together with an MFC sensor while the beam was excited to vibration with the frequency of $17.8 \mathrm{~Hz}$. As a result, according to Fig. 11, the system generated the voltage output until the voltage accumulated on the capacitor of the EHE301 module (Vcap) was lower than $3.7 \mathrm{~V}$.

In the next step, the energy harvesting system parameters were assessed for the vibrating structure by increasing the frequency excitation from 17.8 up to $19.3 \mathrm{~Hz}$. This caused the shift of the slider and temporary switch-off of the generated voltages from the conditioning module. However, at the moment of matching the frequency of the structure vibration to the excitation frequency, the process of the capacitor charging was lunched again. Such behavior of the system led to reappearing of the voltage output from the EH system, which is shown in Fig. 11.

The next step of the experimental setup related assessing the effectiveness of the energy harvesting systems for different sizes of the gap between the slider and the host structure. In order to do this, investigations were carried out for chosen gaps that equaled $0.1 \mathrm{~mm}$ and $0.2 \mathrm{~mm}$, constant resistive load of $R=3.3 \mathrm{k} \Omega$, and constant frequency excitation of $19.3 \mathrm{~Hz}$. As a result, both voltages were measured and then compared with the results obtained for the system with a fixed slider (see Fig. 12).

In the last step, the experimental tests were carried out to compare the effectiveness of both energy harvesting systems connected to the EHE301 module and the SSHI module without a DC-DC system (see Fig. 14). In 

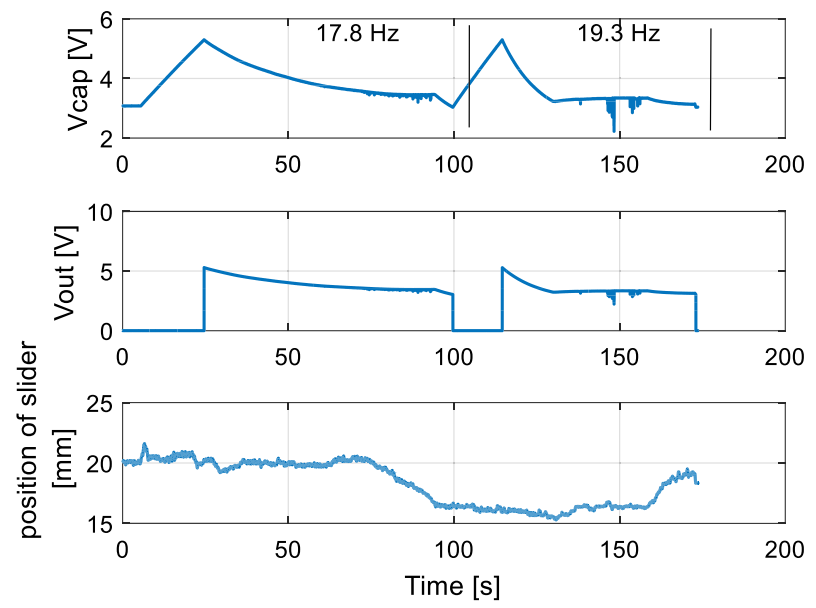

Fig. 11 The influence of the frequency excitation on the behavior of the energy harvesting system
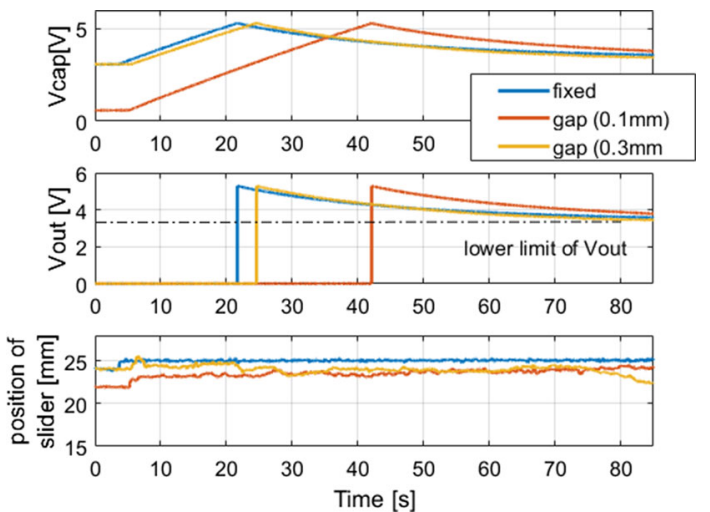

Fig. 12 The comparison of the recorded output voltage, the voltage on the capacitor, and the slider position for the free and the fixed slider on the beam

order to do this, both systems with the gap equaling $0.2 \mathrm{~mm}$ were loaded by the resistive load $R$ of the values of $1 \mathrm{k} \Omega, 3.3 \mathrm{k} \Omega, 10 \mathrm{k} \Omega, 150 \mathrm{k} \Omega, 300 \mathrm{k} \Omega$, and $450 \mathrm{k} \Omega$.

The results of the EH system including the EHE301 module presented in Fig. 13 indicated that this system generally gave poor results due to obtaining low amplitudes of the output voltages $V_{\text {out }}(5.5-6.7 \mathrm{~V})$ and low values of the electrical current $I_{\text {out }}(0.148 \mu \mathrm{A}-0.5 \mathrm{~mA})$ flowing through by these resistive loads $(R=10 \mathrm{k} \Omega$, $R=150 \mathrm{k} \Omega, R=300 \mathrm{k} \Omega, R=450 \mathrm{k} \Omega$ ). As a result, such behavior of the system led to generating also low amplitudes of the output energy $P_{\text {out }}$ in the range $0.1-2.6 \mathrm{~mW}$ and obtaining low effectiveness of this system. In the case of a lower resistive load, this effectiveness may be negligible due to constant charging and discharging of the capacitor. Then, the EH system generates only a periodical output signal whose duration time depends on its value.

In the case of using the EH system with the SSHI module consisting of the inductor $L$ of $4.7 \mathrm{mH}$, four rectifier bridge diodes D1-D4-type D1N4004, the MOSFET transistors BSS138, and the capacitor $100 \mathrm{nF}$ but without a DC-DC converter (LTC 3388 developed by Analog Devices company), significantly better performances were achieved. It was achieved by a PWM signal applied to both transistors and next obtain rapidly changing of the piezoelectric polarity. The photograph of a real module with the electrical scheme is shown in Fig. 14. Such behavior of the system was obtained, especially for higher resistive loads $150 \mathrm{k} \Omega$, $300 \mathrm{k} \Omega$, and $450 \mathrm{k} \Omega$ in which amplitudes of the voltage output signals were reached in the range of 9-30 V, while the electrical currents flowing by the capacitor were close to $400 \mu \mathrm{A}$ (see Fig. 15). As a result, the total output energies that can be generated by this system for the indicated resistive loads are equal to $3.6 \mathrm{~mW}$, $6.4 \mathrm{~mW}, 9.2 \mathrm{~mW}$, respectively. 
(a)

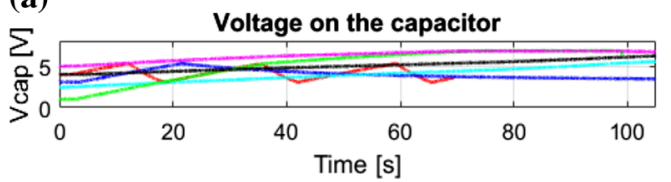

Output voltage

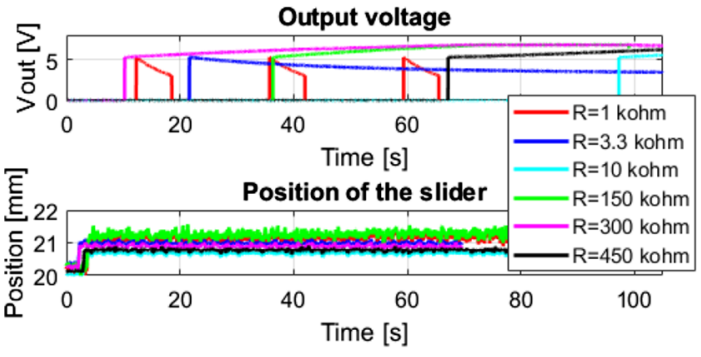

(b)
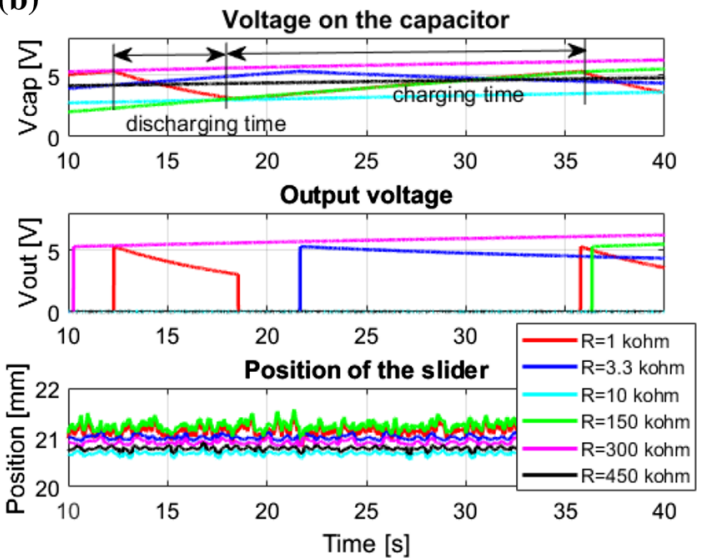

Fig. 13 The comparison of the voltage Vcap accumulated on the capacitor located on the conditioner module EHE301, and the output signal $V_{\text {out }}$ for different values of the resistive load and position of the slider

(a)
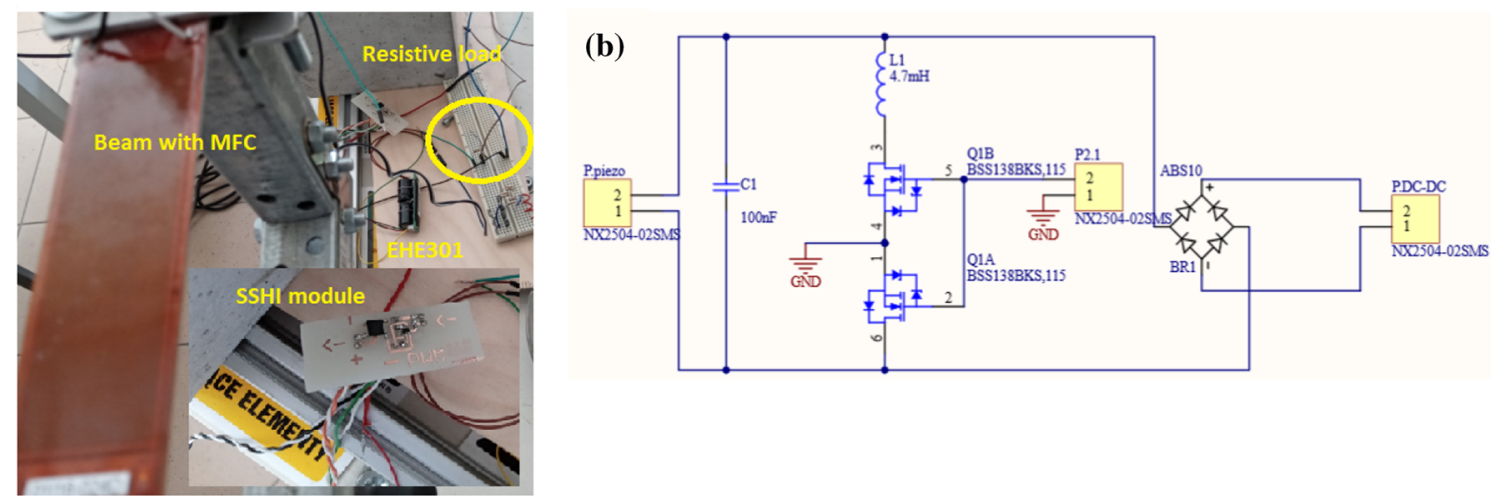

Fig. 14 The SSHI interface designed in Bialystok University of Technology, a a real photograph of the laboratory stand, b the electrical scheme

\section{Summary and conclusions}

As mentioned in Introduction Section, mechanical structures with various boundary conditions and integrated piezo-patches stripes are commonly used in industry and automotive applications to harvest energy from vibrations. As these fields of applications have been developed intensively, such kinds of mechanical structures have been a subject of many articles in the recent two decades. In order to maximize the efficiency of these systems, the problem of the frequency mismatch is being considered by many researchers by using active and passive methods. For this reason, investigations of self-resonance beams with a free sliding mass as a one-dimensional structure are focused on determining distributed parameters of a piezoelectric macro-fiber composite stripe located on a self-resonance beam and checking the possibilities of the considered structure to harvest energy in relation to different values of the slider gap.

For this purpose, as a first step, a modal analysis of the chosen structure with a piezo-patch composite was carried out using the finite element method. As a result, the FEM model consisting of a homogenized model of the MFC element is obtained. The obtained results, presented in Fig. 4, show that the slider location as a proof mass influences the fundamental frequency of the whole structure. This led to detecting two operating regimes for both halves of the beam where a slight movement of the slider generates a significant change of the fundamental frequency value. Further numerical investigations allowed to verify the theoretical results (see Fig. 2) and determine the optimal width of the slider gap in order to harvest the maximum energy amount from vibrations. For this purpose, the voltages generated from the piezo by the strain analysis of the piezo-stripe harvester were analysed for different widths of the gap in the range of $0.1-0.3 \mathrm{~mm}$. The obtained results, 
(a)
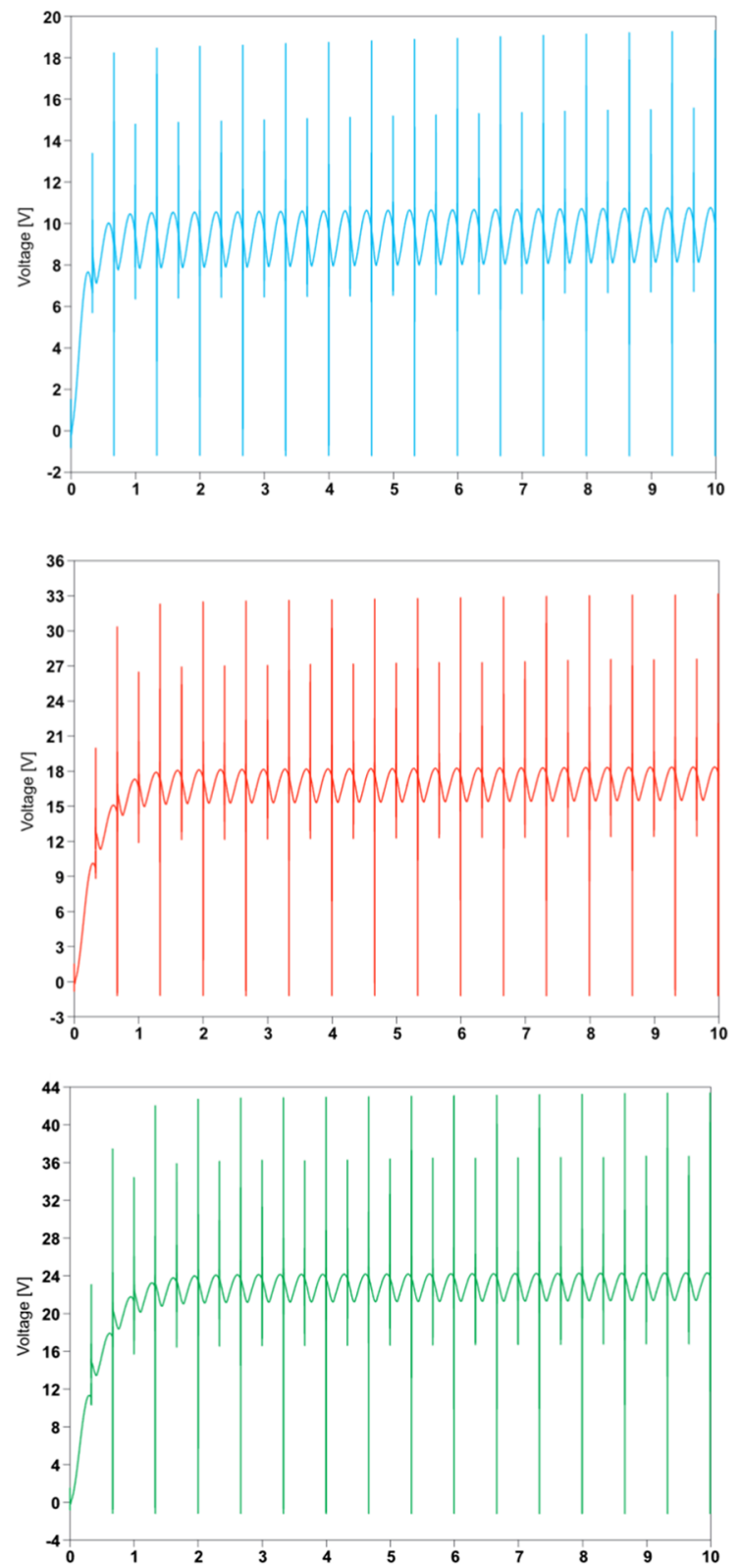

(b)
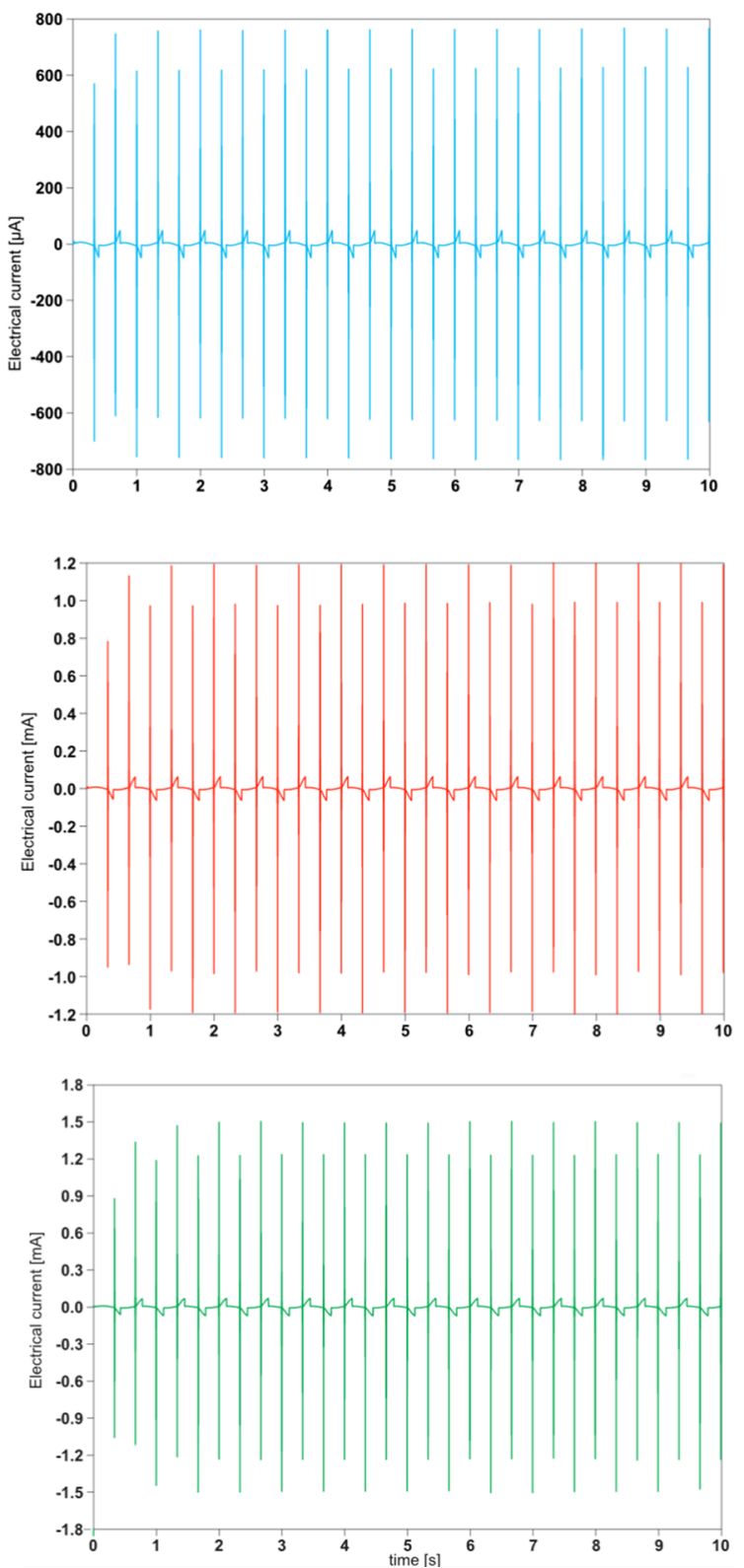

Fig. 15 The recorded signals of $\mathbf{a}$ the voltage on the capacitor, and $\mathbf{b}$ the electrical current flowing through the piezoelectric capacitor by the resistive load of $150 \mathrm{k} \Omega$ (blue line), $300 \mathrm{k} \Omega$ (red line), and $450 \mathrm{k} \Omega$ (green line)

presented in Fig. 7, indicate that increasing the slider gap over 0.2 leads to the decrease in the piezo-harvester strains and results in a decrease in the efficiency of energy harvesting from vibrations.

Experimental investigations of the energy harvesting system carried out on the laboratory stand for a real structure verified properly the numerical results. In the first step, the electromechanical system with a slider was excited to vibration by applying a sinusoidal signal to the vibration shaker. The obtained peaks of the vibration signal from an accelerometer with 5\% uncertainty for the desired location of the fixed slider, presented in Fig. 9b, allowed to detect two operating regimes in both halves of the beam in which the considered system can effectively work in the frequency range of $17-23 \mathrm{~Hz}$. Taking into account the obtained results, the tests were repeated but in the case with the freed slider with the gap of $0.1 \mathrm{~mm}$. Again, the experimental results confirmed the simulations and indicated that the middle part of the beam should be omitted in order to harvest energy from vibrations. 
Further experimental tests allowed to check also the efficiency of the energy harvesting system working in a chosen operating regime for the slider gap of $0.2 \mathrm{~mm}$ during increasing the excitation frequency from 17.8 to $19.3 \mathrm{~Hz}$. The obtained results, presented in Fig. 11, showed that the self-resonance system properly matches the frequency excitation to the indicated position of the slider, which leads to increasing the energy harvesting system efficiency. As a result, this becomes a promising technique for the broadband energy harvesting.

In the last step of the experimental investigations, the voltage signals recorded from both the conditioning systems (EHE301 and SSHI circuit) allowed to assess the effectiveness of the energy harvesting systems for higher resistive loads. The obtained results, presented in Figs. 13 and 14, show that the efficiency of the energy harvesting system consisting of an EHE301 module is low (maximum $2.6 \mathrm{~mW}$ ) due to obtaining low voltage also on the load around $450 \mathrm{k} \Omega$. In the case of the EH system with an SSHI module, it can be observed that the efficiency of this system enhances three times. Then, the output voltage is also higher even for higher restive loads.

Summing up, the performed investigations of an MFC harvester attached to a self-resonating clampedclamped beam enabled assessing the influence of the gap size onto the harvesting performance of the system. The obtained results indicated that the proposed passive method with analyzing the gap size can be a promising technique for the broadband energy harvesting. For this reason, further investigations will be focused on optimal impedance matching ensuring the highest power output from this system and also testing this system by using stochastic signals.

Taking into account the obtained performances of this system, this study may be helpful in designing progressive vibration energy harvesting devices that do not require external power to adjust their resonant frequency to the input vibration. As a result, it can be helpful to develop structural health monitoring of rotating machines in which the phenomenon of rotor unbalancing due to the appearance of not expected vibrations is very dangerous. Then, self-adaptive behavior of this kind of system connected with vibrating structures allows to power small electrical sensors with a low-power demand and detect unbalancing of this machine in an early stage of its performance.

Acknowledgements This work is supported with University Work no. WZ/WM-IIM/1/2019 of Faculty of Mechanical Engineering, Bialystok University of Technology.

Open Access This article is licensed under a Creative Commons Attribution 4.0 International License, which permits use, sharing, adaptation, distribution and reproduction in any medium or format, as long as you give appropriate credit to the original author(s) and the source, provide a link to the Creative Commons licence, and indicate if changes were made. The images or other third party material in this article are included in the article's Creative Commons licence, unless indicated otherwise in a credit line to the material. If material is not included in the article's Creative Commons licence and your intended use is not permitted by statutory regulation or exceeds the permitted use, you will need to obtain permission directly from the copyright holder. To view a copy of this licence, visit http://creativecommons.org/licenses/by/4.0/.

\section{References}

1. Litak, G., Pakrashi, V.: Energy harvesting and applications. Eur. Phys. J. Special Top. 228(7), 1535-1536 (2019)

2. Rafique, S.: Piezoelectric Vibration Energy Harvesting. Modelling and Experiments. Springer, Berlin (2018)

3. Ambrożkiewicz, B., Wolszczak, P., Litak, G.: Modelling of electromagnetic energy harvester with rotational pendulum using mechanical vibrations to scavenge electrical energy. Appl. Sci. 10(2), 671 (2020)

4. Okosun, F., Cahill, P., Hazra, B., Pakrashi, V.: Vibration-based leak detection and monitoring of water pipes using output only piezoelectric sensors. Eur. Phys. J. Special Top. 228(7), 1659-1675 (2019)

5. Koszewnik, A., Oldziej, D.: Performance assessment of an energy harvesting system located on a copter. Eur. Phys. J. Special Top. 228(7), 1677-1692 (2019)

6. Koszewnik, A.: Analytical modeling and experimental validation of an energy harvesting system for the smart plate with an integrated piezo-harvester. Sensors 19(4), 812 (2019)

7. Dal Bo, L., Gardoino, P., Casagrande, D.E., Saggini, S.: Smart panel with sweeping and switching piezoelectric patch vibration absorbers: experimental results. Mech. Syst. Signal Process. 120, 308-325 (2019)

8. Miller, L.M., Halvorsen, E., Dong, T., Wright, P.K.: Modeling and experimental verification of low-frequency MEMS energy harvesting from ambient vibrations. J. Micromech. Microeng. 21, Art. 0450029 (2011)

9. Miller, L.M., Pillatsch, P., Halvorsen, E., Wright, P.K., Yeatman, E.M., Holmes, A.S.: Experimental passive self-tuning behavior of a beam resonator with sliding proof mass. J. Sound Vib. (2013). https://doi.org/10.1016/j.jsv.2013.08.023

10. Krack, M., Aboulfotoh, N., Twiefel, J., Wallaschek, J., Bergman, J., Vakakis, L.A.: Toward understanding the self-adaptive dynamics of a harmonically forced beam with a sliding mass. Arch. Appl. Mech. 87(4), 699-720 (2017). https://doi.org/10. 1007/s00419-016-1218-5

11. Muller, F., Krack, M.: Explanation of the self-adaptive dynamics of a harmonically forced beam with a sliding mass. Arch. Appl. Mech. 90, 1569-1582 (2020) 
12. Yu, L., Tang, L., Yang, T.: Experimental investigation of a passive self-tuning resonator based on a beam-slider structure. Acta Mech. Sin. 45(5), 1079-1092 (2019)

13. Pillatsch, P., Miller, L.M., Halvorsen, L.M.E.: Self-tuning behavior of a clamped-clamped beam with sliding proof mass for broadband energy harvesting. J. Phys. Conf. Ser. 476, 012068 (2013)

14. Greeg, C.G., Pillastch, P., Wright, P.K.: Passively self-tuning piezo-electric energy harvesting system. J. Phys. Conf. Ser. 557, 012123 (2014)

15. Staaf, L.G.H., Smith, A.D., Laundgren, P.: Effective piezoelectric energy harvesting with bandwidth enhancement by asymmetry augmented self-tuning of conjoined cantilevers. Int. J. Mech. Sci. 150, 1-11 (2019)

16. Mori, K., Horibe, T., Ishikawa, S.: Effect of weight on the resonant tuning of energy harvesting devices using giant magnetostrictive materials. Materials 11(581), 1-10 (2018)

17. Firoozy, P., Khadem, S.E., Pourkiaee, M.: Broadband energy harvesting using nonlinear vibrations of a magnetopiezoelastic cantilever beam. Int. J. Eng. Sci. 111, 113-133 (2017)

18. Ducharne, B., Gupta, B., Litak, G.: Simulation of synchronized-switching method energy harvester including accurate piezoceramic nonlinear behavior. Energies 12(4466) (2019)

19. Yan, B., Zhou, S., Litak, G.: Nonlinear analysis of the tristable energy harvester with a resonant circuit for performance enhancement. Int. J. Bifurc. Chaos 28(7), 1850092 (2018)

20. Yan, B., Zhou, S., Litak, G.: Nonlinear analysis of the tristable energy harvester with a resonant circuit for performance enhancement. Int. J. Bifurc. Chaos 28(7), 1850092 (2018)

21. Zhao, H., Wei, X., Zhong, Y., Wang, P.: A direction Self-tuning two-dimensional piezoelectric vibration energy harvester. Sensors 20(77), 1-13 (2020)

22. Ksica, F., Behal, J., Rubes, O., Hadas, Z.: Homogenized model of piezoelectric composite structure for sensing purposes. In: Mechatronics 2019: Recent Advances Towards Industry 4.0, pp. 358-365. Springer (2020)

23. Almizi, M.: Analytical and numerical study of a passive self-tuning resonator. Doctoral thesis, Lund University, Sweden (2018)

24. Koszewnik, A.: Experimental validation of equivalent circuit modelling of the piezo-stripe harvester attached to the SFSF rectangular plate. Acta Mech. et Autom. 14(1), 8-15 (2020)

Publisher's Note Springer Nature remains neutral with regard to jurisdictional claims in published maps and institutional affiliations. 